\title{
Relationship between Body Weight and Self-Esteem: A Study of Young Men and Women in Iran
}

\author{
Seyed Reza Alvani ${ }^{1}$, Seyed Mehrshad Parvin Hosseini ${ }^{\star 2}$ and Laurel Wynne Kimura ${ }^{3}$ \\ ${ }^{1}$ Ph.D in Psychology at, School of social sciences, Unversiti Sains Malaysia, 11800 USM, Pualu Pinang, Malaysia \\ ${ }^{2} \mathrm{Ph} . \mathrm{D}$ in Economics at, Faculity of Bussiness, Multimedia University, 75450 MMU, Malacca, Malaysia \\ ${ }^{3}$ Psychology Lecturer (Ph.D) at, INTI International College, 11900, Pulau Pinang, Malaysia
}

*Corresponding author: Seyed Mehrshad Parvin Hosseini, Ph.D in Economics at, Faculity of Bussiness, Multimedia University, 75450 MMU, Malacca, Malaysia, Tel: 0060124340374, E-mail: mehrshad_ph@yahoo. com

Citation: Seyed Reza Alvani, Seyed Mehrshad Parvin Hosseini, Laurel Wynne Kimura (2016) Relationship between Body Weight and Self-Esteem: A Study of Young Men and Women in Iran. J Obes Overweig 2(2): 202. doi: 10.15744/2455-7633.2.202

Received Date: October 21, 2015 Accepted Date: May 25, 2016 Published Date: May 27, 2016

\begin{abstract}
Background: Obesity and the side effects of weight gain among Iranian females are significantly higher compared to males. In addition, the trend of overweight and obesity among Iranian adolescents in particular is increasing. The objective of this paper was to determine the relationship between body weight status and self-esteem also to evaluate the important role of weight status between the sexes in a conservative society like the Islamic Republic of Iran.

Method: Data were obtained from 450 pre-university students, aged 16-19 years old, in the city of Kashan in Iran. Students were divided based on ascending order into three categories, namely: healthy weight, overweight, and obese. The Rosenberg's Self-esteem Scale was administered to measure level of self-esteem. Body Mass Index was calculated using weight and height measures. The definitions of overweight and obesity were based on age and sex specific BMI (Body Mass Index) cut-off points of the Centers for Disease Control (CDC) and Prevention growth chart 2008. An ordered probit analysis was conducted and the marginal effects of self-esteem and other predictors of self-esteem on body weight status were calculated. Evaluation of gender and self-esteem estimated by a separate binary Probit model.

Results and Conclusion: The results revealed that sex differences and self-esteem were significantly associated with body weight status. Males were at a healthier weight level and had fewer tendencies to be overweight than females. The result of Probit model show that male respondents were less likely to have medium or high self-esteem.
\end{abstract}

Keywords: Obesity; Self-esteem; Weight status; Iran

\section{Background}

Adolescence is an important stage of human development and is characterized by significant somatic growth and maturation of secondary sexual characteristics $[1,2]$.

The World Health Organization (WHO) in a cardiovascular diseases project (MONICA) reported that in 1988, Iran was one of seven countries having the highest prevalence of childhood obesity [3]. Additionally, the trend of overweight and obesity amongst children and adolescence in the Iranian community has been shown to double between the years 1993 and 1999 [4]. Comparable data indicate that in the year 2000, 21.1 per cent and 7.8 per cent of the 11- to 17-year-old adolescents in Tehran were overweight and obese, respectively. According to Ayatollahi's (1992) studies on obesity, between 1988-1989 and 2002-2003, there was evidence of increasing prevalence of obesity from 4.7 per cent to 10.5 per cent among males and 5.1 per cent to 22.5 per cent among females [5]. Consequently, according to the two studies conducted in Iran by Ayatollahi in 1992 and 2010, an increased secular change of 5.8 per cent in men and 17.4 per cent in women emerged over the 18-year gap between the two studies. In other words, obesity and overweight problems are significantly more common among Iranian females compared to males. Furthermore, significant associations between obesity and sex, age, marital status, residential area, and educational level have been found in Iran [6,7].

Increased weight status has been consistently associated with body dissatisfaction among juveniles and adolescents [8,9]. Obesity at certain stages of life, especially adolescence, can damage self-esteem [10], and negative body image is more common among individuals with childhood or adolescent onset of obesity [11]. Although overweight adolescents experience fewer medical complications in comparison to adults, they exhibit some signs of psychological and social distress, including depression and low levels of self-esteem $[12,13]$. 
Concern about body weight has become a common preoccupation in most societies, particularly among females. Unfortunately, the developmental changes associated with puberty tend to move females further away from the current societal prescribed thin beauty ideal rather than closer to it [14]. In different regions of the world, approximately 60 per cent of girls and 30 per cent of boys report a desire to change their size or shape [15,16] and nearly 25 per cent of adolescent females report clinically significant levels of body dissatisfaction [17]. Unfortunately, in Iran, there is relatively little empirical research on body satisfaction and related problems such as emotional distress or any type of physiological or psychological problems. Mifflin, (2007) stated that body dissatisfaction and the desire to be thin is common among Iranian adolescents, and the lifetime prevalence of related disorders like anorexia nervosa, bulimia nervosa and partial syndrome were 0.9 per cent, 3.2 per cent, and 6.6 per cent, respectively [18]. Knowing one's BMI (Body Mass Index) level can help prevent one of the causes of low self-esteem in adolescence, because it signals what could become a contributory factor to obesity and could help families, schools, and health practitioners to begin measures to help children prevent becoming obese.

Sex was found to influence BMI although the direction was not always clear. Ayatollahi and its co-authors (2010) and Rashidypour et al. (2009) argued that there is a significant association between obesity and sex in Iranian society. Parent's education level was also associated with level of BMI in children [19]. The relationship between body image perception and satisfaction from body shape, however, are still disputed.

Harter (1998) found that 25 per cent of adolescent girls were occupied with "how they appear in the eyes of others" [20]. Parents and peers, in particular, can play an important role in influencing the body perception of adolescents [21], and strong evidence exists to demonstrate that obesity can damage self-esteem [10]. Finally, this study argues that family size and level of Cumulative Grade Points Average (CGPA) may influence BMI, a finding that corresponds to prior research [22].

The first goal of this study is to determine the relationship between body weight status and self-esteem from a non-Western perspective, and specifically, in a predominantly Muslim society such as Iran. This led us to evaluate the importance of weight status and body shape between sexes in a conservative society. The second aim of this study is to examine whether sex differences affect body weight status, and if so, at what body weight status (namely, healthy weight, overweight, or obese) and what level of selfesteem (low, medium, or high) does it happen. Other variables could also yield a higher body weight status, such as environmental factors, parents and friends' perceptions, education level, and activities, such as sports.

\section{Methodology}

\section{Participants}

The sample data were captured from a population of pre-university students aged 16-19 years old, in the city of Kashan, Iran. The researcher hired five colleagues to help him to collect participants from the only pre-university center in Kashan city. The questionnaires were distributed to all participants and their height and weight measured at the same time. The sample comprised 225 females and 195 males a total of 450 students.

\section{Instrument}

A 10-item Rosenberg self-esteem scale validated for the Iranian population [23], was utilized to assess global self-worth by measuring both positive and negative feelings about the self. All items were answered using a 4-point Likert scale format, ranging from strongly agree to strongly disagree. After calculating the sum scores of all ten items, we divided them into two categories of high-medium and low. The higher scores, which were 30 to 38, indicated low self-esteem; the lower scores, which were 10 to 29 , indicated high-medium self-esteem ${ }^{1}$. Since the interpretation of dummy variable in the ordered Probit models are easier to explain, Self-esteem value computed based on above and below the sample average into two categories. The variable labeled as low and high-medium and not simply low and high since the levels were divided further in the Table 4 again in order to capture each level of self-esteem on both gender in more detail.

The empirical tests were based on the premise that obesity levels are best viewed through the body mass index (BMI) (Calculated by dividing the square of their height $\left(\mathrm{m}^{2}\right)$ with the value given in units of their weight $\left.(\mathrm{kg}),\left(\mathrm{kg} / \mathrm{m}^{2}\right)\right)$. Following the CDC percentiles growth chart for two to 20- year- olds ${ }^{2}$, in order to capture levels of body weight status, we classified individuals as falling into one of three levels of body image: healthy weight, overweight, or obese.

The present research analyzed body weight status level in terms of BMI. Our evaluation of child and youth obesity followed the categories recommended by Barlow and Dietz (1998) [24] and Barlow (2007) [25], which classified children or teens as overweight if they had BMIs at the $85^{\text {th }}$ to $95^{\text {th }}$ percentiles for age and sex, and as obese if they had BMIs greater than or equal to the $95^{\text {th }}$ percentile. Based on the same studies, a person was in the healthy weight classification if his or her BMI was above the fifth to the $85^{\text {th }}$ percentile as a young adult ${ }^{3}$.

${ }^{1}$ These cut points were calculated based on the mean distribution of the sample. The intention of using mean was to see that on average the self-esteem of the individuals was either medium low or high. This cannot be achieved with computing the median.

${ }^{2}$ Available at www.cdc.gov, accessed on March 6, 2014

${ }^{3}$ These cut points are unchanged from the 1998 expert committee recommendations (Barlow \& Dietz, 1998). 
Hence by inaugurating a new framework for determining the level of body weight status, the current research endeavored to determine the purpose of study by doing an ordered probit analysis. The following socio-demographic and BMI related variables were considered: (1) sex; (2) family education; (3) sport activity; (4) body image perception; (5) body size satisfaction; (6) friend's perception; (7) and self-esteem.

\section{Procedure}

An individual's level of BMI may not be observed directly. The level of BMI (the dependent variable) can be ordered in ascending order from 'healthy weight' to 'overweight' and 'obese'.

Therefore, the dependent variable (level of BMI) coded from 0 for healthy weight students, 1 for overweight students, and 2 for obese students. Since these levels of BMI were ordered in ascending fashion, the ordered probit model was used for the analysis [26,27].

The model can be specified as

$$
y_{n}^{*}=\beta_{1} Z_{1}+\beta_{2} Z_{2}+\beta_{3} Z_{3}+, \ldots . .+Z_{11}+\varepsilon_{n}
$$

Where

$y_{n}^{*}=$ latent and continuous measure of the level of body mass index of students $\mathrm{n}$

$Z_{1-11}=$ a vector of explanatory variables likely to influence BMI. The further explanation of each one is below.

$\beta=$ a vector of parameters to be estimated

$\varepsilon_{\mathrm{n}}=$ a random error term assumed to be normally distributed

The observed and coded discrete level of BMI variable, yn, is determined from the model as follows:

Healthy weight 0 if $-\infty \leq y_{n}^{*} \leq \mu_{1}$

Overweight 1 if $\mu_{1} \leq y_{n}^{*} \leq \mu_{2}$

Obese 2 if $\mu_{2} \leq y_{n}^{*} \leq \infty$

Where

$\mu_{\mathrm{i}}$ 's are thresholds that separate the body image levels; they are estimated along with the vector of parameters.

The BMI-level covariates were sex $\left(Z_{1}\right)$, parent's education $\left(Z_{2} \& Z_{3}\right)$, interest in sports $\left(Z_{4}\right)$, body image perception $\left(Z_{5}\right)$, body satisfaction $\left(Z_{6}\right)$, other people's attitudes towards the individual's body shape $\left(Z_{7} \& Z_{8}\right)$, having a high or medium self-esteem $\left(Z_{9}\right)$, having more than two siblings $\left(Z_{10}\right)$ and having CGPA which falls in first class level category $\left(Z_{11}\right)$. The choice was guided by empirical evidence drawn from similar or broadly related studies elsewhere.

The covariates were defined as follows: a dummy was created for the sex variable, i.e., if the person is male $=1$, and if the person is female $=$ zero. If he or she has a father with a university graduate degree $=1$, and zero if otherwise. A mother with a university graduate degree $=1$, and zero if otherwise. The hypothesis is that the low level of parent's education is associated with higher risk of becoming overweight or obese. The more parents are educated the more they can educate children about their dietary habits. If the person is interested in sport activities $=1$, and zero if otherwise. Body image perception $=1$, and zero if otherwise. If the student is satisfied with his or her physical body shape $=1$, and zero if otherwise. If parents and friends perceive themselves as an obese or overweight person $=1$, and zero if otherwise. If they score high or medium self-esteem $=1$, and zero if the score for self-esteem was low. If the number of siblings is more than two $=1$, and zero if otherwise. Lastly if their CGPA is in first class level category $=1$, and zero if otherwise.

In the following step the relationship between gender and self-esteem were estimated using the simple binary Probit model. The dependent variable, gender for this step were coded 0 for female students, 1 for male students. The sole regressor of the model was categorized as three groups low self-esteem, medium self-esteem and the high self-esteem using the low self-esteem as the reference category.

We can establish the marginal effect of the significant variables (in ordered probit model) on the probability of having higher body weight status. For this, a 'reference person' was needed. It was assumed that the "reference person" in our case was someone who was a female, with both parents not holding a university degree and not interested in sports activities. It was further assumed that she perceives herself as having a healthy weight status but is not fully satisfied with her body shape; parents and friends perceived her as having a healthy weight status; she had relatively low self-esteem; she was a single child or she was from a rather small family; and she was an average student as reflected in her (CGPA) results.

In other word the idea of "holding all other variables constant" is more accurately described as an "independent effect". 


\section{Results}

Table 1 summarizes the descriptive statistics of the study participants. The weights and heights of 450 adolescent students (BMIs) were measured. More than 10 per cent of the total were overweight and about four per cent were obese. Higher percent of female students (57 percent) are having healthy BMI compare to male students (42.41 per cent) with healthy BMI. f the total male students, 42 per cent had a healthy BMI. However, measured against the BMI factor for the total sample, a higher percentage of females were overweight (48 per cent). More than 73 per cent of the obese population was male and only 26 percent were female. The majority of student's at all three levels of BMI were found to have fathers who were educated compared to mothers who were educated. Similarly, more than half of the sample in each of the three levels of our categories exhibited interest in sports activities. About 8 per cent of individuals with healthy BMI perceived themselves as overweight, as opposed to around 48 per cent of the overweight individuals who perceived themselves as having a normal and healthy weight status.

\begin{tabular}{|c|c|c|c|c|}
\hline $\begin{array}{c}\text { Binary Variables Definition codes }(1=\text { if the parameter is in } \\
\text { the category and }(0) \text { if otherwise }\end{array}$ & Healthy & Over Weight & Obese & Total $N=450$ \\
\hline Female & $220(57.0)$ & $30(48.0)$ & $5(26.0)$ & $255(56.6)$ \\
\hline Male & $162(42.41)$ & $19(38.78)$ & $14(73.68)$ & $195(43.33)$ \\
\hline Father with university graduate degree & $60(15.71)$ & $7(14.29)$ & $7(36.84)$ & $74(16.44)$ \\
\hline Mother with university graduate degree & $27(7.07)$ & $4(8.16)$ & $5(26.32)$ & $36(8.00)$ \\
\hline Sport interest (If they are interested in sport activities) & $290(75.92)$ & $39(79.59)$ & $10(52.63)$ & $339(75.33)$ \\
\hline $\begin{array}{c}\text { Body image perception (If they perceive themselves as obese \& } \\
\text { overweight) }\end{array}$ & $31(8.12)$ & $25(51.02)$ & $16(84.21)$ & $72(16.00)$ \\
\hline Body shape satisfaction & $269(70.42)$ & $22(44.90)$ & $3(15.79)$ & $294(65.33)$ \\
\hline Parents perceive them as obese or overweight person & $25(6.54)$ & $23(46.94)$ & $14(73.68)$ & $62(13.78)$ \\
\hline Friends perceive them as obese $\&$ overweight & $21(5.50)$ & $24(48.98)$ & $16(84.21)$ & $61(13.56)$ \\
\hline Self-esteem (If the level of self-esteem is medium or High) & $382(100.00)$ & $32(65.31)$ & $5(26.32)$ & $419(93.11)$ \\
\hline Number of siblings (If they have more than two sibling) & $278(72.77)$ & $34(69.39)$ & $11(57.89)$ & $323(71.78)$ \\
\hline Level of intelligence (If their CGPA is in the first class category) & $127(33.25)$ & $21(42.86)$ & $12(63.16)$ & $160(35.56)$ \\
\hline Sample size (pooled data) & $382(84.89)$ & $49(10.89)$ & $19(4.22)$ & $450(100.00)$ \\
\hline
\end{tabular}

Note: The numbers in parenthesis shows the percentage (\%)

Table 1: Descriptive statistics

A considerable percentage of obese people (15 per cent) did not perceive themselves as obese, and 45 per cent of the overweight participants were satisfied with their body shape, although their parents and friends perceived them as overweight. All of the healthy weight individuals had a self-esteem ranging from medium and to high levels.

The results of the ordered probit analysis are shown in Table 2. The estimated coefficients have no direct interpretation and they only show the effect of independent variables on the dependent variable when coefficients are significant. However, four variables were positive and significantly effecting higher risk of obesity, namely: (1) self- body image perception; (2) parents' perception; (3) friends' perception; and (4) Grade Point Average. Two variables negatively and significantly were affecting body weight status: (1) sex, and (2) self-esteem.

The coefficients for having an educated father and being interested in sport activities in Table 2 shows negative and a not significant effect on the body weight status. Similarly, factors like having an educated mother, being satisfied with their own body shape, and having more than two siblings in the family were weakly effecting body weight status. Although it was expected that showing interest in sports activity would influence BMI level, the sign for this variable was negative and not significant. This may be because merely showing interest in sports activities may not be sufficient, and individuals must actually do some sport activities to reduce their level of BMI. Self-body shape satisfaction had a positive coefficient, although the influence was not strong and the variable was not significant. This is may be because they are not care about self-body shape or maybe because females body shape is not reveling in the public due to Islamic law and must be fully covered.

Entering the characteristics of the "reference person" in estimated ordered probit equation in Table 2, we obtained the log likelihood of (-104.26). The marginal effects that showed in Table 3 exhibit more revealing results since the probabilities of each categories of BMI were recognizable. Since our dependent variable was ordered and divided based on the three types of BMI, Table 3 shows the probabilities of the independent variables for each category of BMI.

A male student in our sample had a higher probability of having a healthy BMI (10.3 per cent) compared to a female student, holding all other variables constant (independent effect). Also probabilities of being overweight for a male student were reduced by -10.3 per cent. The likely ratio of a male being obese was negative ( -0.057 per cent), although it was not significant. 


\begin{tabular}{|c|c|c|c|}
\hline Dependent variable (level of BMI) & Coefficients & Std. Error & P-value \\
\hline Male & $-0.97^{* *}$ & $0.39^{* *}$ & 0.01 \\
\hline Father with university graduate degree & -0.18 & 0.38 & 0.63 \\
\hline Mother with university graduate degree & 0.08 & 0.48 & 0.86 \\
\hline Sport interest (If they are interested in sport activities) & -0.29 & 0.22 & 0.18 \\
\hline $\begin{array}{c}\text { Body image perception (If they perceive themselves as obese \& } \\
\text { overweight) }\end{array}$ & $0.88^{* * *}$ & $0.29^{* * *}$ & 0.002 \\
\hline Body shape satisfaction & 0.23 & 0.27 & 0.39 \\
\hline Parents perceive them as obese or overweight person & $0.52^{* *}$ & $0.26^{* *}$ & 0.04 \\
\hline Friends perceive them as obese \& overweight & $1.17^{* * *}$ & $0.27^{* * *}$ & 0.00 \\
\hline Self-esteem (If the level of self-esteem is medium or High) & $-3.58^{* * *}$ & $0.49^{* * *}$ & 0.00 \\
\hline Number of siblings (If they have more than two sibling) & 0.17 & 0.22 & 0.46 \\
\hline Level of intelligence (If their CGPA is in the first class category) & $0.42^{* *}$ & $0.21^{* *}$ & 0.04 \\
\hline Cut point 1 & -1.61 & 0.57 & \\
\hline Cut point 2 & 0.46 & 0.54 & \\
\hline Observations & 450 & & \\
\hline LR chi ${ }^{*}(11)$ & 254.21 & & \\
\hline Prob > chi ${ }^{2}$ & 0.00 & & \\
\hline Pseudo R & 0.55 & & \\
\hline Log likelihood & -104.26 & & \\
\hline
\end{tabular}

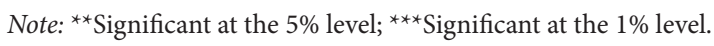

Table 2: Results of the Ordered Probit Estimation

Generally, our results exhibit that female students were at higher risk of being overweight or obese compared to the male students. Students who perceived themselves as obese and overweight regardless of other factors had lower probabilities (-15.31 per cent) of being in the healthy weight category (see Table 3 ).

\begin{tabular}{|c|c|c|c|}
\hline Dependent variable (Body Weight Status) & Healthy & Over Weight & Obese \\
\hline \multirow{2}{*}{ Male } & $10.33^{* * *}$ & $-10.28^{* * *}$ & -0.057 \\
\hline & $(\mathrm{p}<0.001)^{* * *}$ & $(\mathrm{p}<0.001)^{* * *}$ & $(0.371)$ \\
\hline \multirow{2}{*}{ Father with university graduate degree } & 1.82 & -1.81 & -0.007 \\
\hline & $(0.687)$ & $(0.676)$ & $(0.652)$ \\
\hline \multirow{2}{*}{ Mother with university graduate degree } & -0.98 & 0.98 & 0.0046 \\
\hline & $(0.873)$ & $(0.868)$ & $(0.888)$ \\
\hline \multirow{2}{*}{ Sport interest (If they are interested in sport activities) } & 3.65 & -3.63 & -0.02 \\
\hline & $(0.254)$ & $(0.254)$ & $(0.428)$ \\
\hline \multirow{2}{*}{$\begin{array}{c}\text { Body image perception (If they perceive themselves as obese \& } \\
\text { overweight) }\end{array}$} & $-15.31^{* *}$ & $15.15^{* *}$ & 0.16 \\
\hline & $(0.031)^{* *}$ & $(0.034)^{* *}$ & $(0.417)$ \\
\hline \multirow{2}{*}{ Body shape satisfaction } & -2.42 & 2.41 & 0.01 \\
\hline & $(0.347)$ & $(0.387)$ & $(0.572)$ \\
\hline \multirow{2}{*}{ Parents perceive them as obese or overweight person } & -7.83 & 7.78 & 0.06 \\
\hline & $(0.143)$ & $(0.136)$ & $(0.246)$ \\
\hline \multirow{2}{*}{ Friends perceive them as obese \& overweight } & $-24.02^{* * *}$ & $23.63^{* * *}$ & 0.4 \\
\hline & $(0.004)^{* * *}$ & $(0.004)^{* * *}$ & $(0.365)$ \\
\hline \multirow{2}{*}{ Self-esteem (If the level of self-esteem is medium or High) } & $92.60^{* * *}$ & $-55.71^{* * *}$ & $-36.9^{* * *}$ \\
\hline & $(\mathrm{p}<0.001)^{* * *}$ & $(\mathrm{p}<0.001)^{* * *}$ & $(0.005)^{* * *}$ \\
\hline \multirow{2}{*}{ Number of siblings (If they have more than two sibling) } & -1.75 & 1.74 & 0.007 \\
\hline & $(0.474)$ & $(0.444)$ & $(0.565)$ \\
\hline \multirow{2}{*}{ Level of intelligence (If their CGPA is in the first class category) } & -5.21 & 5.19 & 0.027 \\
\hline & $(0.081)$ & $(0.080)$ & $(0.483)$ \\
\hline
\end{tabular}

Note: The numbers are in percentage. The numbers in Parenthesis ( ) shows the P-Value. ${ }^{* *}$ Significant at the $5 \%$ level; $* * *$ Significant at the $1 \%$ level.

$\mathrm{dP} / \mathrm{dx}$ is for discrete change of dummy variable from 0 to 1

Table 3: Marginal Effects (for a discrete change of dummy variables from 0 to 1 ) 
Obviously, participants who perceived themselves as overweight or obese were in fact overweight or obese. In a reverse situation, the odds of being in the overweight category for a person who perceived him/herself as obese or overweight increased by (15.15 per cent). In addition, the chances of the same student falling into the obese category was not significant, but with a positive sign indicating very low chances of the same person being in the obese category by ( 0.16 per cent). However, our ordered probit regression result in Table 2 shows a significant coefficient for the perception of parents in general and all levels of BMI.

Our marginal effect results in Table 3 reveal that there are no significant differences between each of the three categories of body weight status. It can be interpreted that although parents' perception is an important factor influencing BMI level, there is no significant difference among the categories of BMI.

Students whose friends perceived them as obese or overweight were less likely to have healthy BMIs (-24.02 per cent) and more likely to be overweight (23.63 per cent), but they were not significantly more likely to be obese (0.4 per cent).

Interestingly, regardless of other parameters, a student with medium and high self-esteem had the highest probability to be healthy weight (92.60 per cent), and he/she was less likely to be overweight (-55.71 per cent) or obese (-36.9 per cent).

Finally, the results in Table 3 suggest that being a top student reduces the chances of having healthy BMI by -5.2 per cent and increases the chance of being overweight by 5.18 per cent, although topple them into being obese by ( 0.027 per cent) in nonsignificant way. Other factors such as parents' education, sports interest, self-body shape satisfaction, parents' perception, number of siblings, and level of intelligence as measured by CGPA, seem to have rather weak or no significant influence on the different levels of BMI.

As explained before, our reference person was a female. Female students express higher self-esteem compared to male students (Table 3).

So far, our test showed self-esteem is significantly associated to body weight status. In order to explore self-esteem and its relation to sex, a simple probit test was conducted where the sex parameter was situated as the output variable and the levels of self-esteem were the independent variables of the equation. Table 4 demonstrates the results.

The results in Table 4 show that even the different levels of self-esteem are somehow associated with the sex variable. Negative signs of coefficients in Table 4 present the reducing trend of the likelihood ratio. In more detail, the likelihood ratio of being a male student and at the same time having a medium or high self-esteem is less than that for a female student. Interestingly, we can conclude that although a male student is more likely to be healthy weight (according to Table 3 ), he is less likely to have a medium level of self-esteem compared to the referenced female person. The coefficient in Table 4 for male and having high self-esteem does not show any significant relationship, although it had a negative sign.

\begin{tabular}{|c|c|c|c|}
\hline Dependent variable (Gender) & Coefficients & Std. Error & P-value \\
\hline Medium Self-esteem & $-0.61^{* *}$ & $0.26^{* *}$ & $0.02^{* *}$ \\
\hline High Self-esteem & -0.46 & 0.25 & 0.07 \\
\hline
\end{tabular}

Note: ${ }^{*}$ Significant at the $5 \%$ level.

Students with low self-esteem are in the reference category.

Table 4: Results of the Probit Estimation

\section{Conclusion}

The findings of this study generally indicate that individual body image perception, as well as parents' and friends' perceptions, play a significant role in BMI category probabilities, and are supported by other studies such as Maddah \& Nikooyeh, (2010) and Ozmen et al. (2007) [28,29]. Additionally sex and self-esteem are also significantly associated with body weight status. Specifically, our findings concur with those of other studies conducted in Iran $[6,30]$.

Firstly, the current study found that male students have a higher tendency to have a healthy weight as compared to their female counterparts. Secondly, if students perceive themselves as obese or overweight they are less likely to be of healthy weight and more likely to be overweight. These results are in line with prior studies conducted in Iran [3], in the United States [31], in Western Europe [32,33], and in Asian countries such as Japan [34], Korea [35] and China [36]. From the viewpoint of the authors on the base of the results of the present study one of the possible reasons for these outcomes can be that the males are more concerned about their health and so they have lesser tendencies to be overweight. Thirdly, the finding indicates that although female students have a higher tendency to be overweight or obese, they score medium or higher in levels of self-esteem compared to the male students. Several explanations can provide a reason for this phenomenon in Islamic countries. One example is that the compulsory dress codes in Islamic countries for females means that their body shape is not publicly exposed, whereas due to the comparatively relaxed dress codes for males, males tend to be more concerned about their body shape. For females, psychological factors such as lack of positive thinking or exaggeration of their actual weight status cause them to become less interested in keeping in shape $[9,10]$. Although the literature correlating levels of self-esteem and body weight status is scarce $[9,10]$, we found a strong association between self-esteem scores and BMI. In particular, pre-university students had a higher probability of being in the ideal 
ideal status and were less likely to become overweight or obese. These results draw attention to recommendations on society, particularly the school and parents that have an influence on an adolescent's personality and the need for programs in the society for adolescents to have a healthy weight.

The clinical practice of management of weight status should include some programs to increase the client's level of self-esteem. We also found that students with higher levels of GPA were at higher risk of being overweight, which could be due to, students preparing themselves for general university entrance examination while trying to cope with the stress and having insufficient time for workout or leisure.

In conclusion, although further study is required to address these issues, our results indicate that body image perception and peers' perceptions are closely associated with BMI weight categories. The results of this current study also demonstrate how working on adolescent samples can assist countries with a huge work force population in more effectively directing initial resources and research efforts when attempting to control for BMI weight issues. Further study of different nation states could use this framework to elucidate the challenges faced by other societies affecting self-esteem and adolescents, as they are a thriving force in the future development of many states.

\section{Acknowledgement}

We want to thank Prof. Suresh Narayanan, in the school of social sciences, Universiti Sians Malaysia (USM) for his advice and guidance in preparing this paper. Without his assistance, this paper would not have been possible.

\section{References}

1. Malina RM, Bouchard C, Bar-Or O (2004) Growth, Maturation, and Physical Activity (2 ${ }^{\text {nd }}$ Edn), Human Kinetics Publisher, USA.

2. Lobstein T, Baur L, Uauy R (2004) Obesity in Children and Young People: A Crisis in Public Health. Obesity Reviews 5: 4-85.

3. Kelishadi R, Pour MH, Sarraf-Zadegan N, Sadry GH, Ansari R, et al. (2003) Obesity and associated modifiable environmental factors in Iranian adolescents: Isfahan Healthy Heart Program - Heart Health Promotion from Childhood. Pediatr Int 45: 435-42.

4. Kelishadi R, Hashemipour M, Sarraf-Zadegan N, Amiri M (2004) Trend of Atherosclerosis Risk Factors in Children of Isfahan. Asian Cardiovasc Thorac Ann 9: 36-40.

5. Ayatollahi S, Carpenter R (1992) A Study of Adult Height, Weight and Obesity in Shiraz, Iran, 1988-89. Med J Islam Repub 6: 7-12.

6. Ayatollahi S, Ghoreshizadeh Z (2010) Prevalence of Obesity and Overweight among Adults in Iran. Obes Rev 11: 335-7.

7. Rashidy-Pour A, Malek M, Eskandarian R, Ghorbani R (2009) Obesity in the Iranian Population. Obes Rev 10: 2-6.

8. Anschutz DJ, Kanters LJ, Van Strien T, Vermulst AA, Engels RC (2009) Maternal Behaviors and Restrained Eating and Body Dissatisfaction in Young Children. Int J Eat Disord 42: 54-61.

9. Clark L, Tiggemann M (2007) Sociocultural influences and body image in 9- to 12-year-old girls: the role of appearance schemas. J Clin Child Adolesc Psychol 36: 76-86.

10. Griffiths LJ, Parsons TJ, Hill AJ (2010) Self-esteem and quality of life in obese children and adolescents: a systematic review. Int J Pediatr Obes 5: 282-304.

11. Sarwer DB, Wadden TA, Foster GD (1998) Assessment of body image dissatisfaction in obese women: specificity, severity, and clinical significance. J Consult Clin Psychol 66: 651-4.

12. Neumann C (1983) Obesity in Childhood Developmental-Behavioral Pediatrics, Saunders Publishers, Philadelphia, USA, 536-51.

13. Mendelson BK, White DR (1982) Relation between body-esteem and self-esteem of obese and normal children. Percept Mot Skills 54: 899-905.

14. Robins RW, Trzesniewski KH, Tracy JL, Gosling SD, Potter J (2002) Global self-esteem across the life span. Psychol Aging 17: 423-34.

15. Ricciardelli LA, McCabe MP (2001) Children's body image concerns and eating disturbance: a review of the literature. Clin Psychol Rev 21: 325-44.

16. Wood KC, Becker JA, Thompson JK (1996) Body Image Dissatisfaction in Preadolescent Children. J Appli Dev Psychol 17: 85-100.

17. Stice E, Whitenton K (2002) Risk factors for body dissatisfaction in adolescent girls: a longitudinal investigation. Dev Psychol 38: 669-78.

18. Mifflin H (2007) The American Heritage Medical Directory: Orlando (FL): Houghton Mifflin Company Publisher, USA.

19. Lamerz A, Kuepper-Nybelen J, Wehle C, Bruning N, Trost-Brinkhues G, et al. (2005) Social class, parental education, and obesity prevalence in a study of sixyear-old children in Germany. Int J Obes 29: 373-80.

20. Harter S (1998) The Development of Self-Representations. In: Social, Emotional, and Personality Development, Volume 3, Handbook of Child Psychology $\left(5^{\text {th }}\right.$ Edn), Wiley Publishers, USA.

21. Stice E (1994) Review of the Evidence for a Sociocultural Model of Bulimia Nervosa and an Exploration of the Mechanisms of Action. Clin Psychol Rev 14: 633-61.

22. Parsons TJ, Power C, Logan S, Summerbell CD (1999) Childhood predictors of adult obesity: a systematic review. Int J Obes Relat Metab Disord 23: S1-107.

23. Shapurian R, Hojat M, Nayerahmadi H (1987) Psychometric Characteristics and Dimensionality of a Persian Version of Rosenberg Self-Esteem Scale. Perceptual and Motor Skills 65: 27-34.

24. Barlow SE, Dietz WH (1998) Obesity evaluation and treatment: Expert Committee recommendations. The Maternal and Child Health Bureau, Health Resources and Services Administration and the Department of Health and Human Services. Pediatr 102: E29.

25. Barlow SE (2007) Expert Committee Recommendations Regarding the Prevention, Assessment, and Treatment of Child and Adolescent Overweight and Obesity: Summary Report. Pediatrics 120: S164-92.

26. Tan AK, Yen ST, Feisul MI (2012) Determinants of Body Weight Status in Malaysia: An Ethnic Comparison. Int J Public Health 57: 279-88. 
27. Greene W (2004) The behaviour of the maximum likelihood estimator of limited dependent variable models in the presence of fixed effects. Econometrics J 7: $98-119$.

28. Maddah M, Nikooyeh B (2010) Factors associated with overweight in children in Rasht, Iran: gender, maternal education, skipping breakfast and parental obesity. Public Health Nutr 13: 196-200.

29. Ozmen D, Ozmen E, Ergin D, Cetinkaya AC, Sen N, et al. (2007) The association of self-esteem, depression and body satisfaction with obesity among Turkish adolescents. BMC Public Health 7: 80.

30. Moayeri H, Bidad K, Aghamohammadi A, Rabbani A, Anari S, et al. (2006) Overweight and obesity and their associated factors in adolescents in Tehran, Iran, 2004-2005. Eur J Pediatr 165: 489-93.

31. Schwartz MB, Brownell KD (2004) Obesity and Body Image. Body Image 1: 43-56.

32. Sánchez-Villegas A, Madrigal H, Martínez-González MA, Kearney J, Gibney MJ, et al. (2001) Perception of body image as indicator of weight status in the European union. J Hum Nutr Diet 14: 93-102.

33. Gualdi-Russo E, Albertini A, Argnani L, Celenza F, Nicolucci M, et al. (2008) Weight status and body image perception in Italian children. J Hum Nutr Diet 21: $39-45$

34. Inoue M, Toyokawa S, Miyoshi Y, Miyano Y, Suzuki T, et al. (2007) Degree of agreement between weight perception and body mass index of Japanese workers: MY Health Up Study. J Occup Health 49: 376-81.

35. Kim DS, Kim HS, Cho Y, Cho SI (2008) The effects of actual and perceived body weight on unhealthy weight control behaviors and depressed mood among adult women in Seoul, Korea. J Prev Med Public Health 41: 323-30.

36. Xie B, Liu C, Chou CP, Xia J, Spruijt-Metz D, et al. (2003) Weight perception and psychological factors in Chinese adolescents. J Adolesc Health 33: 202-10.

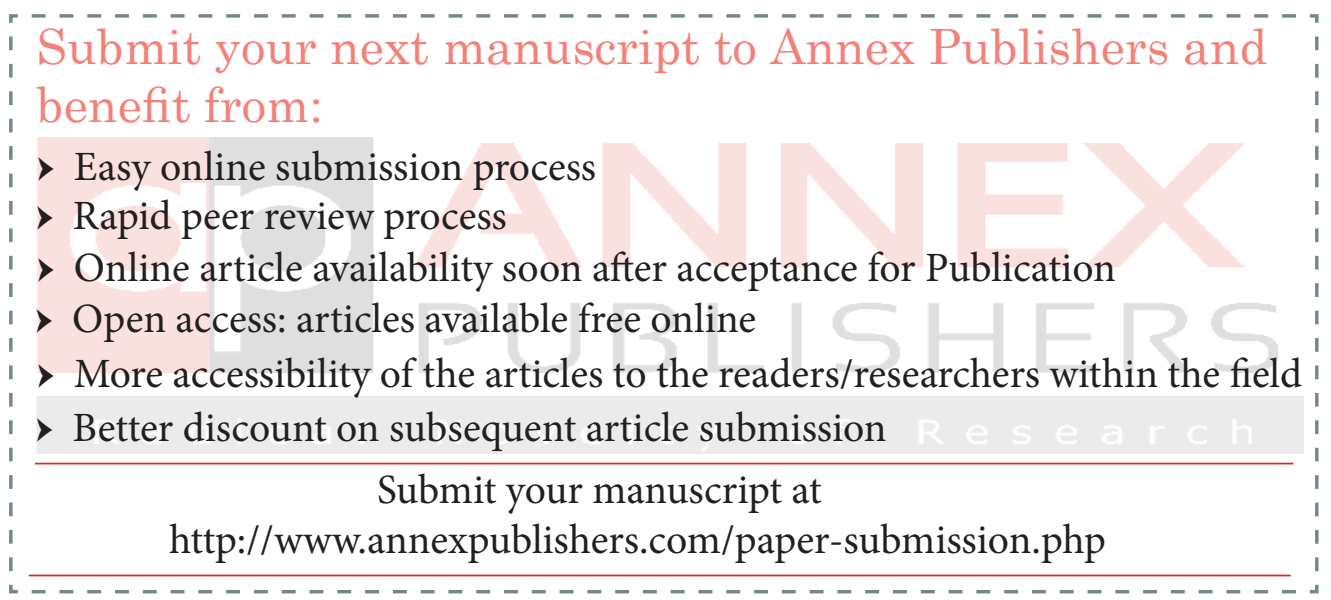

